\title{
基于卷积神经网络与时频图纹理信息的 信号调制方式分类方法
}

\author{
白艺远，许华，孙莉
}

(空军工程大学 信息与导航学院, 陕西 西安 710077)

\begin{abstract}
摘 要: 通信信号的调制方式识别是通信侦察、频谱监测的重要工作内容之一,提出一种利用深度学 习提取信号时频图纹理信息的分类方法。该方法利用不同调制方式在时频图细节上的微弱差别, 并 使用卷积神经网络提取图像纹理特征, 最终输入 SOFTMAX 分类器进行分类。结果表明,该方法在大 样本条件下, 可取得良好的分类效果。与传统基于特征参数的支持向量机分类方法或前馈神经网络 方法相比, 其提取特征更优、分类效果更好,同时减少了人工设计特征参数的工作量和不确定性。
\end{abstract}

关 键 词: 调制识别; 时频图纹理信息; 深度学习; 卷积神经网络 中图分类号:TN92

文献标志码:A

文章编号: 1000-2758(2019)04-0816-08

由于在通信侦察、频谱监测等领域中的重要作 用, 通信信号调制方式的自动识别一直是通信信号 处理的主要研究内容之一。特别是复杂电磁环境下 的调制方式自动识别,由于受到通信信号种类繁多、 信噪比低、信道衰落及信号自适应变化等影响,其识 别效果还无法满足实用要求。目前除了对卫星通信 的信号监测应用以外, 国内外尚无完全满足应用需 要的无线电信号自动识别和分类系统。因此, 在现 有研究成果的基础上, 研究探索应用新技术提升复 杂电磁环境下通信信号自动识别和分类的性能具有 重要意义。

当前,信号分类问题广泛采用基于特征的方 法 $^{[1-6]}$, 即首先对信号的时域、频域信息进行特征参 数提取, 随后利用神经网络 ${ }^{[1-3]}$ 、支持向量机等 ${ }^{[4-6]}$ 分类器进行分类。近年来, 利用信号间统计似然特 征的差异进行分类的最大似然方法也逐步得到重 视 ${ }^{[7-9]}$ 。基于特征的方法需要根据所判别的信号种 类采用实现最佳可分性的特征值, 其最优特征随着 信道变化而不同, 人工选择工作量大; 同时, 所处理 的信号内部可能存在特定的联系, 这些深层次特征 不易被参数化描述。基于最大似然方法在使用时需 要完整的信道先验信息, 但实际情况中往往难以取
得；同时，该方法须假设信号先验符合均匀分布 ${ }^{[5]}$, 局限性大。近年来, 深度学习技术作为神经网络技 术的最新发展,在图像、语音识别领域获得了广泛应 用,其核心思想在于使用新的算法、网络结构提升神 经网络的泛化能力, 基于深度学习的神经网络能够 自动进行特征学习, 由于实现了分层特征抽取, 反映 了数据本身的结构, 深度学习在大多数场景下表现 优于手工特征提取。基于此,本文研究利用信号时 频图对通信调制信号进行分类, 将通信信号分类问 题转化为图像分类问题, 并使用基于深度学习技术 的卷积神经网络取代浅层学习结构, 通过卷积核对 二维数据的纹理信息与空间结构进行特征提取, 捕 捉不同调制方式在时频图上的微小差异, 从而实现 信号分类。

\section{1 基于时频图差异的信号调制方式 分类}

当前, 基于人工设计特征参数和浅层学习的信 号分类方法获得了广泛应用，即通过人工分析并确 定信号分类所需要的特征参数, 将信号映射至特征 
空间,再使用 SVM、ANN 等分类器进行处理。但是, 基于信号瞬时幅度、频率、相位信息的识别方法易受 信道的影响,在低信噪比下效果差; 而基于高阶统计 量的处理方法难以区分 QAM 信号, 必须借助循环 谱进一步处理, 而循环谱的运算量大, 目前还难以实 时计算 $^{[10]}$ 。

时频图分析是一种基于短时傅里叶变换 (STFT) 的常用人工辅助通信信号分析手段, 即通过 观察、判别时频图在频点、包络和结构上的差异区别 各类信号。时频图能够反映非平稳信号时间、频率 和功率之间的关系, 其中时间和频率的最小分辨率 组合为一个矩形, 称之为时频原子。但部分调制方 式的差异难以通过图像结构信息体现,不能利用时 频图直接观察得到。图 1a) 的 2FSK 信号存在 2 条 高能量谱线, 易于识别, 但图 1b)、图 1c) 中的 QPSK、8PSK 信号则难以观察区分, 这是由于人眼观 察时频图时, 总是更为注意时频图在结构、轮廓上的 差异, 而高阶调制的频带掩盖了时频原子的潜在分 布差异, 难以体现信号内部变化规律。

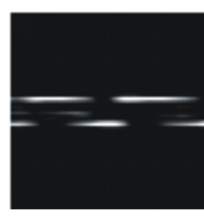

a) 2FSK 信号

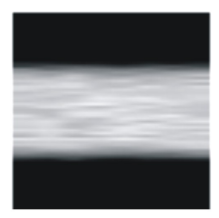

b) QPSK 信号

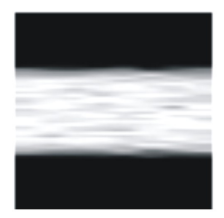

c) 8 PSK 信号
图 1 不同调制方式时频图图样

深度学习中的卷积神经网络 ( CNN) 能够学习、 利用图像在多个尺度上的基本结构, 这一特性与时 频图的多尺度特性匹配。因而, 使用 CNN 对时频图 的纹理和结构关系进行学习, 就能够确定信号所属 的调制方式, 为探讨利用纹理信息分类的可行性, 本 文首先利用灰度共生矩阵 ${ }^{[11]}$ 、图像局部二值模 式 ${ }^{[12]}$ 来描述时频图的纹理信息, 并说明了这些图像 特征与信号特征的映射关系。

\section{1 基于时频图特征参数分类方法及其局限性}

1 ) 灰度共生矩阵

灰度共生矩阵反映了图像灰度分布关于方向、 变化幅度和局部领域的综合信息 ${ }^{[13]}$ 。其定义为在 $\theta$ 方向上, 相隔距离 $d$ 的一对像素分别具有灰度值 $i$ 和 $j$ 出现的概率, 记为 $P(i, j, d, \theta)$, 利用灰度共生矩 阵可以得到 4 类特征参数:

(1) 角二阶矩 $C_{1}$

$$
C_{1}=\sum_{i=0}^{L-1} \sum_{j=0}^{L-1}[P(i, j, d, \theta)]^{2}
$$

式中, $L$ 为图像最大灰度,下同。

(2) 惯性矩 $C_{2}$

$$
C_{2}=\sum_{i=0}^{L-1} \sum_{j=0}^{L-1}(i-j)^{2} P(i, j, d, \theta)
$$

(3) 逆差分矩 $C_{3}$

$$
C_{3}=\sum_{i=0}^{L-1} \sum_{j=0}^{L-1}\left[\frac{P(i, j, d, \theta)}{1+(i-j)^{2}}\right]
$$

(4) 熵 $C_{4}$

$$
C_{4}=\sum_{i=0}^{L-1} \sum_{j=0}^{L-1} P(i, j, d, \theta) \lg [P(i, j, d, \theta)]
$$

2) 图像局部二值模式

图像局部二值模式( LBP) 是一种用于描述图像 局部纹理特征的方法 ${ }^{[14-15]}$ 。设图像 $I$ 尺寸为 $(a, b)$, $I$ 上有点 $P(x, y)$, 在以 $P$ 为中心的 $3 \times 3$ 窗口内, 以 $P$ 灰度为阈值, 将另外 8 个像素的灰度值与 $P$ 进行比 较, 若灰度值大于 $P$ 灰度值, 则该点被标记为 1 , 否 则为 0 。这样, $3 \times 3$ 窗口内的 8 个点经比较可产生 8 位二进制数, 即得到该窗口中心像素点的 LBP 值, 并最终生成一个 $(a-1, b-1)$ 大小的 LBP 谱, 其值 域为 $0 \sim 255$ 。

本文选择 $d$ 取 $[1,4], \theta$ 取 $0^{\circ}, 45^{\circ}, 90^{\circ}, 135^{\circ}$ 下 的灰度共生矩阵统计量, 和图像 LBP 谱直方图构成 联合特征用于分类。由于联合特征维数为 320 , 因 此使用了稀疏自编码器 (SA) 进行降维, 得到了 128 维的联合特征,它保留了原 320 维特征的全部信息。

表 1 基于时频图纹理特征的分类实验结果准确率 \%

\begin{tabular}{cccc}
\hline \multirow{2}{*}{ 信号种类 } & \multicolumn{3}{c}{ 信噪比/dB } \\
\cline { 2 - 4 } & 2 & 4 & 6 \\
\hline 256QAM, 1 024QAM & 69.3 & 74.0 & 73.8 \\
QPSK, 8PSK & 55.2 & 43.4 & 46.3 \\
2FSK, MSK & 89.2 & 96.5 & 99.7 \\
\hline
\end{tabular}

从实验中可以看到, 对于 $2 \mathrm{FSK}, \mathrm{MSK}$ 信号, 基 于时频图纹理特征的分类方法有良好的效果,但对 QPSK,8PSK 和 256QAM, 1 024QAM 信号则不能分 辨。本文认为原因在于:

1) 图像的特征统计量不能反映图像特征的组 合特性。对于 LBP 而言,如图像纹理具有较多的特 征模式 $x$,则 LBP 直方图 $x$ 对应二进制数处会出现 较高的峰值, 该峰值仅仅显示了 $x$ 的统计特性, 对于 
该特征出现的位置、特征间的组合关系则不能体现， 同理灰度共生矩阵也存在相同的问题。

2) 模型维数过高。由于不能事先确定最为有 效的特征参数,算法保留了全部特征,可能导致冗余 信息对最优特征构成稀释。

在图像处理中,纹理体现的是物体在空间分布 的规律及相关性 ${ }^{[16]}$, 人工设计的纹理特征参数具有 对其空间意义的描述,而时频谱图中的纹理特征是 时间序列映射至高维空间的信息奇异性表征,而利 用灰度信息的纹理特征提取方法难以在高维空间对 其描述 ${ }^{[17]}$, 且易受噪声影响, 因而效果较差。因此, 本文采用基于卷积神经网络的深度学习方法, 通过 高维空间抽取来获取信号的纹理特征,再通过降维 实现不同调制信号的分类。

\section{2 卷积神经网络}

卷积神经网络 (CNN) 是一种专门用于处理具 有矩阵结构数据的神经网络, 其与普通神经网络的 主要区别在于 $\mathrm{CNN}$ 使用由卷积层和池化层(降采样 层) 构成的特征提取器, 每个卷积层包含多个特征 图,特征图内的单元共享权值以实现参数稀疏 ${ }^{[18]}$ 。 $\mathrm{CNN}$ 模仿人眼处理信息的分层方式, 利用卷积操作 以获取不同尺度卷积核对图像某一区域的激活响 应, 实现分层特征提取, 从而使网络反映数据的本质 结构。

研究表明, 图像可以分为由不同方向线性函数 所描述的边缘 ${ }^{[11-12]}$, 将这些二维线性函数组成的矩 阵称为图胞,如图 2 所示。

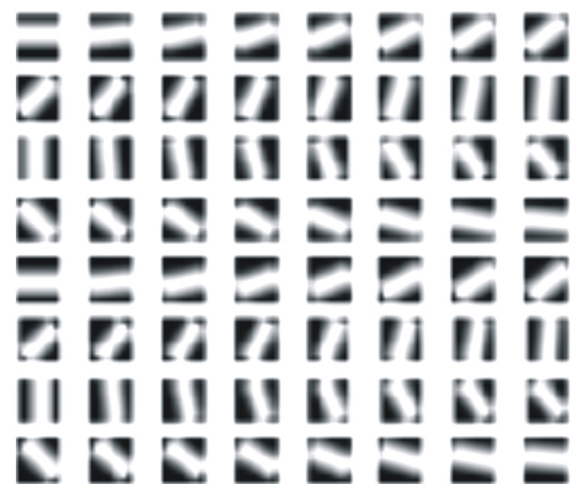

图 2 由 Gabor 权值描述的图胞

对任意一个图胞 $P$, 将其视为坐标 $(x, y)$ 的函 数, 记为 $P(x, y)$, 则任意一个复杂二维图像 $I$ 可表示 为各图胞的加权和, 记权重为 $w(x, y)$, 则有

$$
I=\sum_{x} \sum_{y} w(x, y) P(x, y)
$$

一般情况下, $w(x, y)$ 具有 Gabor 函数的性质, 此时 另记 $w(x, y)$ 为

$$
\begin{aligned}
& w\left(x, y ; \alpha, \beta_{x}, \beta_{y}, f, \phi, x_{0}, y_{0}, \tau\right)= \\
& \quad \alpha \exp \left(-\beta_{x} x^{\prime 2}-\beta_{y} y^{\prime 2}\right) \cos (f x+\phi)
\end{aligned}
$$

式中

$$
\begin{gathered}
x^{\prime}=\left(x-x_{0}\right) \cos \tau+\left(y-y_{0}\right) \sin \tau \\
y^{\prime}=-\left(x-x_{0}\right) \sin \tau-\left(y-y_{0}\right) \cos \tau
\end{gathered}
$$

$\alpha, \beta_{x}, \beta_{y}, f, \phi, x_{0}, y_{0}, \tau$ 为参数,这些图胞是图像在不 同尺寸上的切分的组成元素, 即小尺度特征的加权 和构成大尺度特征。这些大尺度特征同样可以构成 更大尺度的特征,从而形成多层结构。根据图像切 分得到的特征不是依靠人工确定的算法得到的特征 值, 而是图像本身的结构信息, 因而能够学习时频图 纹理信息的最优特征 ${ }^{[19]}$,如图 3 所示。

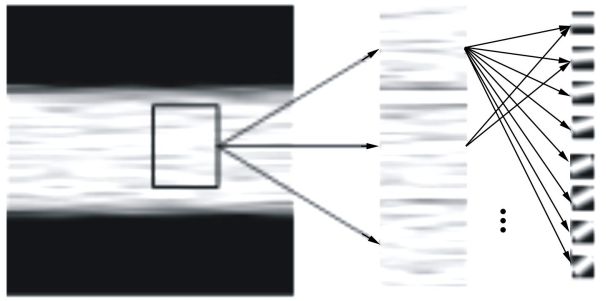

图 3 图像分层切分示意

具体学习方法为:

步骤 1 随机生成卷积核及各卷积核权值, 并 使用这些核对图像进行二维卷积操作, 可以得到图 像某一区域对卷积核的激活值, 随后将激活值通过 ReLU 激活函数处理, 以避免传统网络结构可能出 现的梯度消失问题;

步骤 2 对激活值进行池化,即降采样, 从而赋 予卷积核对于微小形变、噪声的不变性,降采样后的 激活值作为下一层的输人。

步骤 3 将分类输出误差利用反向传播分摊至 各层权值, 进行调整, 最终完成各个尺度上卷积核权 值的训练。

\section{3 识别算法设计}

\subsection{1 信号分类方法}

本节利用卷积神经网络提取通信信号时频图纹 理特征,并使用 SOFTMAX 分类器进行分类, 分类使 用欧氏距离损失函数 


$$
e=\sum(l-y f(x))^{2}
$$

式中, $l$ 为信号样本的输出, $y$ 为样本标签, 其具体步 骤为:
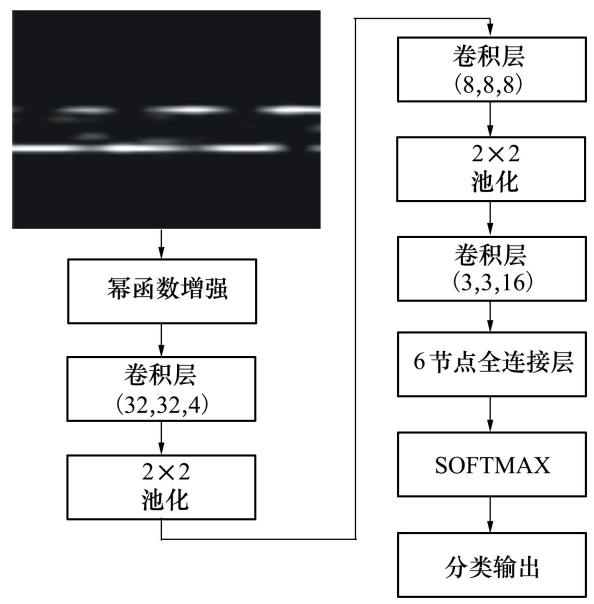

图 4 信号识别算法流程

步骤 1 时频图生成与标记。训练开始时, 利 用信号样本生成时频图, 并根据对应的调制方式进 行标记。为减少 STFT 带来的纹波, 将时频图进行 自适应幂函数增强 ${ }^{[20]}$, 使得其带外部分能量降至频 带内-30 dB 以下。

步骤 2 无监督的图像特征提取。使用随机初 始化的卷积核检测图像的纹理,构成特征。

步骤 3 有监督的参数微调。将网络输出接人 SOFTMAX 分类器, 得到所属类别, 并与信号标签进 行对比, 将误差向后传递并调整权值。误差下降至 满足终止条件时, 在测试集验证网络准确性, 即可得 到目标网络。

\subsection{2 神经网络参数确定}

目前学界普遍认为 $\mathrm{CNN}$ 卷积核的大小决定了 CNN 的特征提取能力, 因而必须确定卷积核的大小 使其能够观察到图像在纹理、结构上的区别。本文 认为小尺度卷积核应当能够覆盖时频原子, 使得一 个小尺度卷积核匹配一个 FFT 单元,下面对其尺寸 与 FFT 参数进行分析, 试图确定其大小。

假设样本信号 $S(t)$ 采样率为 $F_{s}$, 码元速率为 $R_{b}$, 时长为 $T$, 则总长度为 $T \cdot F_{s}$ 点; 在进行 $n$ 点长 STFT 后生成时频图长度为 $\frac{T \cdot F_{s}}{n}$ 点, 每个时频原子 包含 $\frac{R_{b} \cdot n}{F_{s}}$ 个码元; 在奈奎斯特采样下, 最高频率为 $\frac{F_{s}}{2}$, 频率分辨率为 $\frac{F_{s}}{n}$, 则共有 $\frac{n}{2}$ 点; 最终形成时频图
的尺寸为 $\left(\frac{T \cdot F_{s}}{n}, \frac{n}{2}\right)$, 随后将其缩放至 $(L, L)$, 假设 卷积核按层由上到下大小分别为 $(x, x),(y, y),(z$, $z)$ 。本文中, 为限制问题复杂度, 确定以 3 个时频原 子组成一个卷积核, 即 $z$ 取 3 , 此时有 $3^{M \frac{R_{b} \cdot n}{F_{s}}}$ 种时频原 子组合, 将其视为 16 个卷积滤波器加权和, 即卷积 核参数为 $(3,3,16)$ 。

其他 2 层参数确定尚无数学根据, 现由多次实 验确定 $x$ 取 $32, y$ 取 8 。第一层共 4 个过滤器, 第二层 为 8 个,第三层 16 个。

\section{2 实验验证}

为验证方法的有效性, 本文设计了包含 $2 \mathrm{FSK}$, MSK, QPSK, 8PSK, 256QAM , 1 024QAM 6 种调制方 式在内的六分类实验, 观察分类效果, 并借助卷积核 梯度图像分析核特性。

\section{1 信号样本设计}

实验中为去除其他因素的影响, 采用随机生成 的 0,1 比特, 经调制生成采样率 $F_{s}$ 为 $10 \mathrm{MHz}$ 、符号 速率 $R$ 为 $1 \mathrm{M}$ 波特的信号, 信号共计 10000 点, FFT 窗长取 1024 。

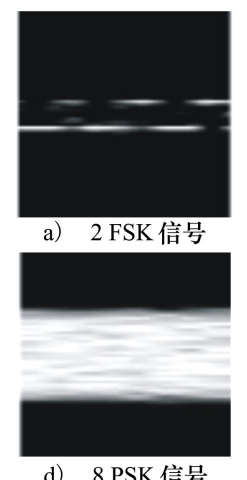

d） 8 PSK 信号

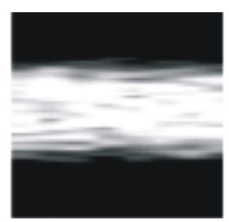

b） MSK 信号

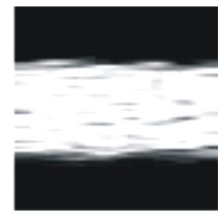

e) 256 QAF 信号

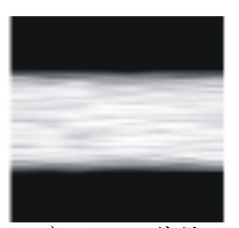

c) QPSK 信号
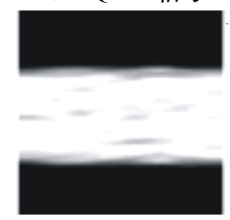

f) 1024 QAM 信号
图 5 时频图样本

对 于 2FSK, MSK, QPSK, 8PSK, 256QAM, $1024 \mathrm{QAM}$ 信号, 其载频 $F_{c}$ 取 $2 \mathrm{MHz}$, 第一零点带宽 $2 \mathrm{MHz}, 2 \mathrm{FSK}$ 信号的 $F_{c_{1}}$ 取 $2.2 \mathrm{MHz}, F_{c_{2}}$ 取 $1.8 \mathrm{MHz}$, 并生成时频图, 每组随机数对应生成 6 个时频图样 本, 并全部缩放至 $128 \times 128$, 共生成 24000 个样本, 如图 5 所示, 图 5a) 5f) 分别为 2FSK, QPSK, 8PSK, MSK, 256QAM, 1 024QAM 信号。从中取3 600个样 本作为测试集, 另外 20400 个样本作为训练集。 


\section{2 调制样式分类实验}

本文在实验中, 将训练集信号样本组成尺寸为 $(128,128,20400)$ 的矩阵导人神经网络, 待网络训 练完毕后对测试集中的图像进行分类测试。实验共 进行 5 次, 结果取平均, 保留 1 位小数。为体现效 果,这里同时采用文献 $[2-4]$ 方法作为对比。

文献 [2]中, 使用信号的奇异谱、功率谱、小波 能量谱熵特征作为特征参数输人 SVM 进行分类。

文献 [3] 中,使用了信号的高阶原点矩和高阶 累积量构成 17 维联合参数, 输人多层感知机 (MLP) 进行分类, 该 MLP 共 3 层, 单元数分别为 $20,8,6$ 。

文献 [4] 中, 将信号高阶统计量 $\left|C_{61}\right|^{2} /\left|C_{42}\right|^{3}$ 和平滑后的频谱相关峰值作为特征参数, 输人 SVM 分类器进行分类。

为体现图像非线性增强的有效性, 同时对比增 强前后效果, 记录准确率如表 2 所示。可以看到, 本 文算法具有良好的分类能力, 在进行幂函数增强且 信噪比为 $0 \mathrm{~dB}$ 时,仍能得到 $89.1 \%$ 的准确率, 较基 于特征值的方法提升约 7\%, 而在信噪比为 $6 \mathrm{~dB}$ 以 上时准确率可达 $99 \%$, 且本文算法仅涉及对信号中 心频率的估计, 减少了人工设计特征参数的工作量。

表 2 分类准确率数据

$\%$

\begin{tabular}{|c|c|c|c|c|c|c|}
\hline \multirow{2}{*}{ 方法 } & \multicolumn{6}{|c|}{ WK 信噪比/dB } \\
\hline & 0 & 2 & 4 & 6 & 8 & 10 \\
\hline 熵特征-SVM ${ }^{[2]}$ & 68.4 & 71.2 & 79.7 & 81.9 & 87.4 & 88.6 \\
\hline 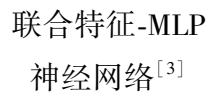 & 61.2 & 65.8 & 70.1 & 76.4 & 84.1 & 90.1 \\
\hline $\begin{array}{c}\text { 高阶统计量- } \\
\text { 循环谱-SVM } \\
\text { 算法 }^{[4]}\end{array}$ & 81.4 & 85.7 & 92.2 & 98.7 & 100 & 100 \\
\hline 本文算法 & 89.1 & 92.2 & 97.0 & 99.0 & 100 & 100 \\
\hline $\begin{array}{c}\text { 本文算法(无幂 } \\
\text { 函数增强) }\end{array}$ & 82.3 & 83.9 & 92.1 & 97.6 & 99.1 & 100 \\
\hline
\end{tabular}

一般而言, 神经网络的学习过程难以观察, 为了 解释网络学习成果, 本文采用梯度图像诊断网络参 数, 即导出网络中某个给定层对于输人图像的梯度, 随后将其缩放, 使其与输人大小保持一致并与输人 图像叠加, 再次作为输人; 如此反复迭代多次, 使得 最终输出反映该核的特性。
图 6 为卷积层 1 生成梯度图像。由于卷积层 1 的核尺寸较大,因而卷积运算对图像形成平滑,弱化 了纹理的影响, 主要关注信号在频谱图中的位置。 可以看到,卷积层 1 获取了信号高能量部分 (图像 中心）。
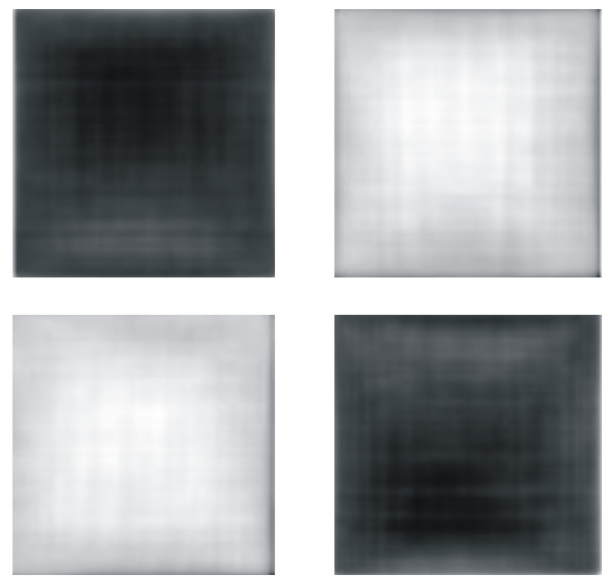

图 6 卷积层 1 生成梯度图像
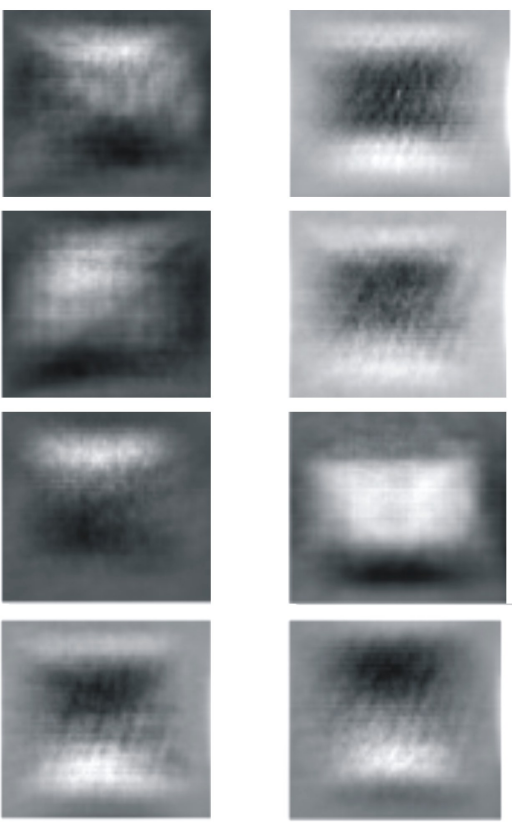

图 7 卷积层 2 生成梯度图像

卷积层 2 尺寸减小,使得各滤波器进一步反映 了信号结构信息。从图 7 中可以看到,该层提取了 时频图的上下边缘，由于时频图不是恒包络的，因而 边缘不平整, 另外, 由于卷积核较大, 该层仍然不能 提取纹理信息。

随着卷积核进一步减小至能够与时频原子大小 相比拟时, 能够反映图像时频原子的特性, 观察提取 
的纹理信息可以发现,生成图像的高能量部分存在 偏移,即卷积核对中频偏移不敏感。

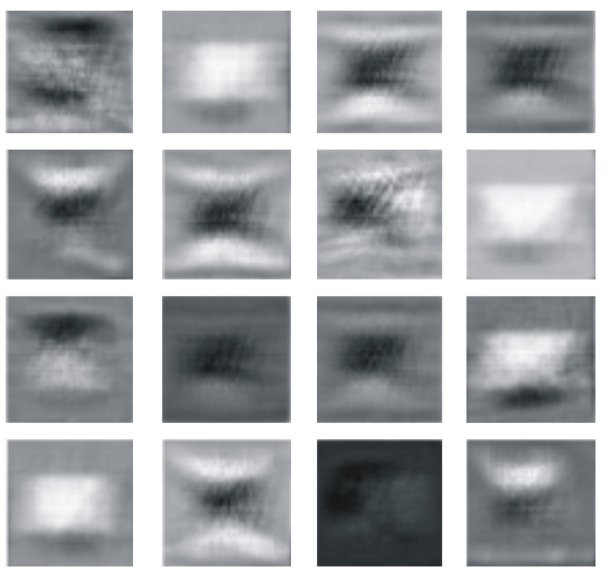

图 8 卷积层 3 生成梯度图像

图 9 为全连接层生成梯度图像, 全连接层获取 与其匹配的纹理, 并将其放大, 可以观察到图像中低 能量部分存在简单图案的重复, 这是由卷积核激活 值最大的部分生成图像叠加在输人的低能量区上形 成的图案, 因而消除了输人信号能量的影响, 换言 之, 即神经网络认为各类时频图是由这些简单图案 构成的, 可以认为神经网络成功提取了纹理特征, 并 成功区分了这些从时频图结构特征上无法区分的信 号类型。

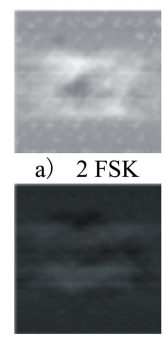

d) 8 PSK
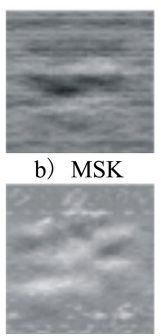

e) $256 \mathrm{QAM}$
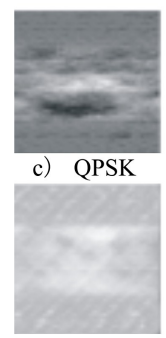

f) $1024 \mathrm{QAM}$
图 9 全连接层生成梯度图像

\section{3 扩展类别识别}

为了验证方法对更多调制方式的适应性, 本文 还进行了包含 $2 \mathrm{FSK}, 4 \mathrm{FSK}, \mathrm{MSK}, \mathrm{BPSK}, \mathrm{QPSK}$, 8PSK, 8QAM , 16QAM , 64QAM , 256QAM , 1 024QAM 信号在内共计 11 类信号的调制识别实验。

由于高阶 PSK 调制的抗干扰性能较差, 实际应 用中很少使用 16 及以上进制的 PSK 调制, 出于同 样的原因也不使用 1024 以上进制的 QAM 调制。 在实验之前, 可以联想到 8QAM 和 $8 \mathrm{PSK}$ 的星座图
接近, 各相位上的幅值差别较小, 当在 8 个相位上具 有相同的符号映射时难以分清, 因此首先进行 8QAM 和 8PSK 类别分类实验以验证适应性。信号 参数、神经网络网络设计同 2.1 节一致, 2 类各提供 4000 个时频图样本作为训练集, 另外各 200 个作为 测试集。测试结果如下 (保留 1 位小数, 实验进行 5 次, 结果取平均值)。

表 3 本文算法分类准确率数据

\begin{tabular}{ccccccc}
\hline 信噪比 $/ \mathrm{dB}$ & 0 & 2 & 4 & 6 & 8 & 10 \\
准确率 $/ \%$ & 49.9 & 53.2 & 58.9 & 64.2 & 71.8 & 75.4 \\
\hline
\end{tabular}

可以看到二分类结果较差, 算法不能有效区分 8QAM 和 8PSK 信号。但考虑到 8QAM 和 8PSK 信 号解调方法没有大的区别, 接下来的实验中将把 $8 \mathrm{QAM}, 8 \mathrm{PSK}$ 作为一类进行处理。对于 $2 \mathrm{FSK}$, 4FSK, MSK, BPSK, QPSK, ( 8PSK, 8QAM ), 16QAM, 64QAM, 256QAM, 1 024QAM 共计 10 类信号, 信号 参数、神经网络设计同 2.1 节一致, 每类各提供 10 000个样本作为训练集, 另外各 200 个作为测试 集。测试结果如下。

表 4 分类准确率数据

$\%$

\begin{tabular}{|c|c|c|c|c|c|c|}
\hline \multirow{2}{*}{ 方法 } & \multicolumn{6}{|c|}{ 信噪比/dB } \\
\hline & 0 & 2 & 4 & 6 & 8 & 10 \\
\hline 熵特征-SVM ${ }^{[2]}$ & 54.6 & 59.9 & 65.7 & 71.2 & 80.4 & 87.3 \\
\hline $\begin{array}{c}\text { 联合特征-MLP } \\
\text { 神经网络 }^{[3]}\end{array}$ & 55.2 & 57.3 & 66.7 & 73.1 & 79.5 & 86.9 \\
\hline $\begin{array}{c}\text { 高阶统计量- } \\
\text { 循环谱-SVM } \\
\text { 算法 }^{[4]}\end{array}$ & 63.2 & 67.5 & 71.5 & 77.5 & 81.4 & 89.6 \\
\hline 本文算法 & 71.2 & 76.2 & 80.7 & 91.9 & 94.5 & 94.9 \\
\hline $\begin{array}{c}\text { 本文算法(无幂 } \\
\text { 函数增强) }\end{array}$ & 72.1 & 79.3 & 83.3 & 90.5 & 93.8 & 94.0 \\
\hline
\end{tabular}

结果表明, (1) 在分类数量增加时, 分类性能出 现了一定下降,算法仍能保持精确度在 $70 \%$ 以上。 (2) 在信噪比小于等于 $4 \mathrm{~dB}$ 时, 幂函数增强不起作 用, 此时应当关闭增强特性。 


\section{3 结 论}

本文针对信号调制方式分类问题中基于特征参 数的机器学习方法的缺点, 提出利用信号时频图将 一维信号分类问题转化为二维图像分类问题, 解释 了二维图像特征参数和一维信号特征参数之间的映 射关系, 并针对基于特征参数的图像分类对信号时 频图的不适应性, 提出使用 CNN 提取图像特征, 将 特征参数反映在网络参数内, 并将其用于信号分类, 实验中, 对 2FSK, MSK, QPSK, 8PSK, 256QAM, 1 024QAM 信号的分类取得了较好的结果, 证明了其
相对传统方法的优越性。下一步的工作中, 可以对 神经网络结构进一步调整, 使其具有识别训练集以 外样本的能力, 由于数字调制种类有限, 因而可以尝 试利用信号的进制数、频点数、中心频率、频偏等特 征参数的集合 $\boldsymbol{G}$ 完成对某一信号调制方式的语义 描述,这个语义描述可以代替人工标注的标签。此 时, 可以将神经网络定义为 3 层模型, 第一层为本文 所描述的 3 层 $\mathrm{CNN}$, 第二层为一个 $n$ 参数的分类 器, 用于判断 $\mathrm{CNN}$ 输出和 $\boldsymbol{G}$ 的对应关系, 第三层为 一个语料库, 根据 $n$ 个参数将信号映射到具体类别, 从而实现在无样本情况下的零样本语义识别。

\section{参考文献:}

[1] 陈红,蔡晓霞,徐云, 等. 基于多重分形特征的通信调制方式识别研究 $[\mathrm{J}]$. 电子与信息学报, 2016, 4(38): 863-869 CHEN Hong, CAI Xiaoxia, XU Yun, et al. Communication Modulation Recognition Based on Multi-Fractal Dimension Characteristics $[\mathrm{J}]$. Journal of Electronics and Information Technology, 2016, 38(4) : 863-869 (in Chinese)

[2] 杨发权,李赞,李红艳, 等. 基于蜂群算法和神经网络的通信调制识别方法 [J]. 系统工程与电子技术, 2013, 35(10): 2186-2191

YANG Faquan, LI Zan, LI Hongyan, et al. Research of Communication Modulation Recongnition Based on Bee Colony Algorithm and Neural Netowork [J]. Systems Engineering and Electronics, 2013, 35(10): 2186-2191 (in Chinese)

［3］李一兵,葛娟,林云. 基于熵特征和支持向量机的调制识别方法 $[\mathrm{J}]$. 系统工程与电子技术,2012, 34(8)：1691-1695

LI Yibing, Ge Juan, LIN Yun. Modulation Recongition Using Entropy Features and SVM[J]. Systems Engineering and Electronics, 2012, 34(8): 1691-1695 (in Chinese)

[4] 赵雄文, 郭春霞, 李景春. 基于高阶累积量和循环谱的信号调制方式混合识别算法 [ J ] . 电子与信息学报, 2016, 38(3): $674-680$

ZHAO Xiongwen, GUO Chunxia, LI Jingchun. Mixed Recognition Algorithm for Signal Modulation Schemes by High-Order Cumulants and Cyclic Spectrum[J]. Journal of Electronics \& Information Technology, 2016, 38(3): 674-680 (in Chinese)

[5] 韩洁, 张涛,王欢欢, 等. 基于 3D-Hibert 能量谱和多尺度分形特征的通信辐射源个体识别 [ J ]. 通信学报, 2017, 38(4): 99-109

HAN Jie, ZHANG Tao, WANG Huanhuan, et al. Communication Emitter Individual Identification Based on 3D-Hibert Energy Spectrum and Multi-Scale Fractal Features[J]. Journal on Communications, 2017, 38(4) : 99-109 (in Chinese)

[6] NANDI A K, AZZOUZ E E. Algorithms for Automatic Modulation Recognition of Communication Signals [J]. IEEE Trans on Communications, 1998, 46(4): 431-436

[7] 付俊强, 李蓉,赵成林, 等. 基于贝叶斯序贯推理的自适应调制识别算法 [ J ] . 系统工程与电子技术, 2015, 37 (12): 2860-2864

FU Junqiang, LI Rong, ZHAO Chenglin, et al. Sequential Bayesian Inference Based Adaptive Modulation Recognition Algorithm [J]. Systems Engineering and Electronics, 2015, 37(12) : 2860-2864 (in Chinese)

[8] 刘明骞,李兵兵, 曹超凤, 等. 认知无线电中非高斯噪声下数字调制信号识别方法 $[\mathrm{J}]$. 通信学报, 2014, 35( 1) : 82-88 LIU Mingqian, LI Bingbing, CAO Chaofeng, et al. Recognition Method of Digital Modulation Signals over Non-Gaussian Noise in Cognitive Radio[J]. Journal on Communications, 2014, 35(1): 82-88 (in Chinese)

[9] 陆珊珊,王伟,王国玉. 幅相调制信号的星座图恢复与调制方式识别 [J]. 国防科技大学学报,2016, 38(6)：130-134 LU Shanshan, WANG Wei, WANG Guoyu. Constellation Recovery and Modulation Recongition for Multiplequadrature Amplitude Modulation Signals[J]. Journal of Mational University of Defense Technology, 2016, 38(6) : 130-134 (in Chinese) 
[10] SWAMI A, SADLER B M. Hierarchical Digital Modulation Classification Using Cumulants $[\mathrm{J}]$. IEEE Trans on Communications, $2000,48(3): 416-429$

[11] HARALICK R, SHANMNGAM K, DINSTEIN I. Texture Feature for Image Classification[J]. IEEE Trans on Systems, Manand Cybernetics, 1973, 3(6): 768-780

[12] 冀中,聂林红. 基于抗噪声局部二值模式的纹理图像分类 $[\mathrm{J}]$. 计算机研究与发展, 2016,53(5): 1128-1135

JI Zhong, NIE Linhong. Texture Image Classification with Noise-Tolerant Local Binary Pattern $[\mathrm{J}]$. Journal of Computer Research and Development, 2016, 53(5): 1128-1135 (in Chinese)

[13] LEE H, GROSSE R, Ranganath R. Convolutional Deep Belief Networks for Scalable Unsupervised Learning of Hierarchical Representations [C] // International Conference on Machine Learning, 2009

[14] LEE Y J, GRAUMAN K. Learning the Easy Things First: Self-Paced Visual Category Discovery[C] // Conference on Computer Vision and Pattern Recognition, 2011

[15] NIXON M, ALBERTO S. Aguado. Feature Extraction and Image Processing[M]. Amsterdam, Elsevier Academic Press, 2008

[16] KRIZHEVSKY A, SUTSKEVER I, HINTON G. Imagenet Classification with Deep Convolutional Networks [C] // Advances in Neural Information Processing Systems, 2012: 1097-1105

[17] GOODFELLOW I, COURVILLE A. On Distinguishability Criteria for Estimating Generative Models[C] // International Conference on Learning Representations, 2014

[18] KINGMA D, REZENDE D, MOHAMED S. Semi-Supervised Learning with Deep Generative Models [C] // Neural Information Processing Systems, 2014

[19] BERTONCINI C, RUDD K, NOUSAIN B. Wavelet Fingerprinting of Radio-Frequency Identification(RFID) Tags [ J]. IEEE Trans on Industrial Electronics, 2012, 59(12) : 4843-4850

[20］孙延奎. 小波分析及其应用 $[\mathrm{M}]$. 北京:机械工业出版社, 2005

SUN Yankui. Wavelet Analysis and Application[M]. Beijing, Mechanical Industry Press, 2005 (in Chinese)

\title{
A Recognition Algorithm for Modulation Schemes by Convolution Neural Network and Spectrum Texture
}

\author{
BAI Pengyuan, XU Hua, SUN Li \\ (Institute of Information and Navigation, Air Force Engineering University, Xi'an 710077, China)
}

\begin{abstract}
The recognition of modulation schemes for communication signals is an important part of communication surveillance and spectrum monitoring. An algorithm based on deep learning and spectrum texture is proposed to recognize modulation schemes. Based on imperceptible differences among various spectrums of modulation schemes, the algorithm uses Convolution Neural Network to capture the features of image texture and thus classify the features with a SOFTMAX classifier. The experiment shows the algorithm performs better than traditional algorithm based on feature parameters, while the features captured can better reveal the signal detail and reduces effort on feature parameter design.
\end{abstract}

Keywords : modulation classification ; spectrum texture; deep learning; convolution neural network ; algorithm 